\title{
Overexpression of Survivin and XIAP in MDR cancer cells unrelated to $\mathbf{P}$-glycoprotein
}

\author{
ZHI SHI ${ }^{1,2}$, YONG-JU LIANG ${ }^{1}$, ZHE-SHENG CHEN ${ }^{2,3}$, XIAO-HONG WANG ${ }^{1}$, \\ YAN DING $^{1}$, LI-MING CHEN ${ }^{1}$ and LI-WU FU ${ }^{1,3}$ \\ ${ }^{1}$ State Key Laboratory for Oncology in South China, Cancer Center, Sun Yat-Sen University, \\ Guangzhou 510060, P.R. China; ${ }^{2}$ Department of Pharmaceutical Sciences, College of \\ Pharmacy and Allied Health Professions, St. John's University, Jamaica, NY 11439, USA
}

Received November 30, 2006; Accepted January 29, 2007

\begin{abstract}
Cancer cells developing multidrug resistance (MDR) is one of the most serious clinical problems responsible for the failure of cancer chemotherapy. P-glycoprotein (P-gp) overexpression and inhibitor of apoptosis proteins (IAPs) overexpression in cancer cells are the two common mechanisms of MDR. However, the relationship between IAPs and P-gp in MDR cancer cells is unknown. We investigated the expression levels of two IAPs, Survivin and XIAP, and their interaction with P-gp in MDR cancer cells. We have found that the human epidermoid carcinoma cells $\mathrm{KBV}_{200}$ and breast cancer cells MCF-7/Adr overexpress not only P-gp but also XIAP and Survivin, and showed high resistance to chemotherapeutic drugs doxorubicin, docetaxel and vincristine, in contrast to their parental cells KB and MCF-7. Furthermore, upregulation of Survivin or XIAP through transfection with the plasmid pECFPN1-Survivin or pcDNA3-6myc-XIAP in these four cell sublines did not affect the P-gp expression. Downregulation of Survivin or XIAP through transfection with the Survivin or XIAP siRNA did not have an effect on the P-gp expression in these resistant cells. Additionally, our immunoprecipitation results showed that Survivin or XIAP did not directly bind to
\end{abstract}

Correspondence to: Dr L.-W. Fu, State Key Laboratory for Oncology in South China, Cancer Center, Sun Yat-Sen University, Guangzhou 510060, P.R. China

E-mail: Fulw@mail.sysu.edu.cn

Dr Z.-S. Chen, Department of Pharmaceutical Sciences, St. John's University, Jamaica, NY 114309, USA

E-mail: Chenz@stjohns.edu

Abbreviations: MDR, multidrug resistance; P-gp, P-glycoprotein; ABC, ATP-binding cassette; IAPs, inhibitor of apoptosis proteins; XIAP, X-linked IAP; DMEM, Dulbecco's modified Eagle's medium; RNAi, RNA interference; siRNA, small interfering RNA; MTT, 3-(4,5-dimethylthiazol-yl)-2,5-diphenyltetrazolium bromide; PVDF, polyvinylidene fluoride

Key words: MDR, P-gp, IAPs, Survivin, XIAP, tumor
P-gp. In summary, our study suggested that the overexpression of Survivin and XIAP in MDR cancer cells does not directly interact with P-gp.

\section{Introduction}

Cancer cells developing resistance to chemotherapeutic drugs is a frequent clinical problem encountered in the treatment of human cancers. After obtaining resistance to a single drug or a class of drugs, cancer cells show cross-resistance to other functionally and structurally unrelated drugs which include the anthracyclines, vinca alkaloids, taxanes, topoisomerase I inhibitors, and other natural products. This phenomenon is known as multidrug resistance (MDR) which is one of the most serious problems responsible for the failure of cancer chemotherapy (1). Various cellular mechanisms result in MDR, including increased drug efflux, alteration in cell cycle checkpoints, failure of apoptotic mechanisms, changes in targeted enzymes, altered DNA repair and scavenging enzymes $(2,3)$. Among these mechanisms of MDR, the most common and extensively studied mechanism is cancer cells overexpressing P-glycoprotein (P-gp), an ATP-binding cassette $(\mathrm{ABC})$ transporter, which is a $170-\mathrm{kD}$ plasma membrane glycoprotein encoded by the MDR1 gene acting as a drug efflux pump to remove chemotherapeutic drugs from cancer cells, and its expression level decides the magnitude of resistance. Additionally, it has been suggested that, aside from the drug efflux pump, P-gp might also confer resistance to apoptosis through specific mechanisms by inhibiting the activation of the cell death effector enzymes caspases from different stimuli, such as anticancer drugs, serum starvation, UV or $\gamma$ irradiation, and ligation of the cell surface death receptors Fas and tumor necrosis factor receptor (4-6). Nevertheless, this inhibitory effect of P-gp might be reversed by using specific P-gp antagonists, such as anti-P-gp antibodies or the pharmacological inhibitor verapamil $(4,5)$.

The inhibitor of apoptosis proteins (IAPs), which are able to inhibit apoptosis induced by a variety of stimuli, are always overexpressed in cancer cells that give rise to resistance to apoptosis. IAPs are a class of structurally related proteins that were initially identified in baculoviruses. At present, eight human IAPs have been identified: c-IAP-1, c-IAP-2, ILP-2, 
neuronal apoptosis inhibitory protein (NAIP), X-linked IAP (XIAP), Survivin, Livin, and Apollon/Bruce (7). IAPs are defined by one or more repeats of a highly conserved $\sim 80$ amino-acid zinc-binding domain located at the amino-terminus, termed the baculovirus IAP repeat domain. It has been shown that IAPs block apoptosis either by binding and inhibiting specific caspases, or through caspase-independent mechanisms (7). In addition, IAPs exhibit other biological functions that include involvement in protein degradation (8), and c-IAP1 and c-IAP2 are integral parts of the type-2 TNF-receptor complex (9), while Survivin plays a role in cell mitosis, mainly in microtubule organization (10). Recently, several studies showed that the MDR leukemia cells HL-60R significantly overexpressed not only P-gp but also both the mRNAs and all the IAPs studied, i.e. c-IAP-1, c-IAP-2, XIAP, NAIP and survivin except for c-IAP-1 mRNA, and their expression levels of IAPs were much less affected by the treatments with chemotherapeutic drugs in comparison with their parent sensitive cells HL-60 $(11,12)$. The overexpression of either P-gp or IAPs in cancer cells can lead to MDR, and MDR cancer cells may overexpress P-gp and/or IAPs. Here, we speculated if there are interactions between P-gp and IAPs during the process of MDR cancer cells developing resistance to apoptosis induced by various stimuli.

In this study, we examined the protein expression of Survivin and XIAP, the two most important and studied IAP family members, in the human epidermoid carcinoma cells KB, breast cancer cells $\mathrm{MCF}-7$, and their resistant cells $\mathrm{KBV}_{200}$ and MCF-7/Adr, and explored the interactions between XIAP, Survivin and P-gp in these two resistant cells.

\section{Materials and methods}

Materials. RPMI-1640 medium, Dulbecco's modified Eagle's medium (DMEM), OPTI-MEM medium, fetal bovine serum and Lipofectamine 2000 were purchased from Invitrogen Life Technologies Inc. (Carlsbad, CA, USA). Doxorubicin was purchased from Hisun Pharmaceutical Co., and vincristine was purchased from Main Luck Pharmaceuticals Inc. Docetaxel and 3-(4,5-dimethylthiazol-yl)-2,5-diphenyltetrazolium bromide (MTT) were purchased from Sigma Chemical Co. (St. Louis, MO, USA). The plasmid pECFPN1-Survivin was kindly provided by Dr Marko J. Kallio (VTT-Medical Biotechnology and University of Turku, Finland), and the plasmid pcDNA36myc-XIAP was kindly presented by Dr Robert G. Korneluk (University of Ottawa, Canada). The plasmid pcDNA3 was purchased from Invitrogen Inc., and the plasmid pECFPN1 was purchased from Clontech Co. (Mountain View, CA, USA). Mouse monoclonal anti-human Hsp70 antibody, rabbit polyclonal anti-human P-gp antibody, goat polyclonal anti-human Survivin and XIAP antibodies, HRP-conjugated anti-mouse, anti-rabbit, anti-goat IgG, and Protein A/G plus-agarose were purchased from Santa-Cruz Biotechnology Inc. (Santa Cruz, CA, USA). Mouse monoclonal anti-human P-gp antibody was purchased from Calbiochem Inc. (La Jolla, CA, USA). Rabbit polyclonal anti-human Survivin and XIAP antibodies, and a Chemiluminescent Western Detection kit were purchased from Cell Signaling Technology Inc. (Beverly, MA, USA). The Plasmid Midi kit was purchased from Qiagen Inc. (Valencia, CA, USA).
Cell lines and cell culture. $\mathrm{KBv}_{200}$ cells, human epidermoid carcinoma MDR cells expressing a high level of P-gp which is the main inducer of MDR, were cloned from parental drugsensitive KB cells by stepwise exposure to increasing doses of vincristine and ethylmethane sulfonate mutagenesis. $\mathrm{KBv}_{200}$ cells were $\sim 60$-fold resistant to vincristine in comparison with KB cells. These two cells were obtained from Professor Y.S. Liu (Chinese Academy of Medical Sciences, Beijing). MCF-7/Adr cells were classical human breast adenocarcinoma MDR cells cloned from parental drug-sensitive MCF-7 cells through stepwise exposure to increasing doses of doxorubicin and similarly overexpressed P-gp. MCF-7/Adr cells were approximately 100 -fold resistant to doxorubicin in comparison with MCF-7 cells. MCF-7/Adr and MCF-7 cells were generously provided by Professor X.Y. Liu (National Cancer Institute, USA). All four cell lines were grown as adherent monolayers in flasks in RPMI-1640 or DMEM culture medium with $10 \%$ fetal bovine serum, benzylpenicillin $(50 \mathrm{U} / \mathrm{ml})$, and streptomycin $(50 \mathrm{ng} / \mathrm{ml})$ at $37^{\circ} \mathrm{C}$ in a humidified atmosphere of $5 \% \mathrm{CO}_{2}$.

MTT cytotoxicity assay. Cells were harvested with trypsin and resuspended in a final concentration of $2 \times 10^{4}$ cells $/ \mathrm{ml}$ respectively. Aliquot $(190 \mu \mathrm{l})$ for each cell suspension was distributed evenly into 96-well multiplates. After cells were incubated for $24 \mathrm{~h}$, designated wells were treated with different concentrations of doxorubicin, docetaxel or vincristine $(10 \mu \mathrm{l} /$ well). After 68 h, 3-(4,5-dimethylthiazol-yl)-2,5-diphenyltetrazolium bromide (MTT) solution $10 \mu 1(5 \mathrm{mg} / \mathrm{ml})$ was added to each well, and the plate was further incubated for $4 \mathrm{~h}$ at $37^{\circ} \mathrm{C}$ in $5 \% \mathrm{CO}_{2}$, allowing viable cells to reduce the yellow MTT into dark-blue formazan crystals which were dissolved in $100 \mu 1$ dimethylsulfoxide. The absorbance in individual wells was determined at 540 and $655 \mathrm{~nm}$ by a microplate Reader (Bio-Rad). The inhibition of cell growth was evaluated by the MTT method by using triplicate assay. The concentrations required to inhibit growth by $50 \%$ ( $\mathrm{IC}_{50}$ values) were calculated from cytotoxicity curves by Bliss method.

siRNA. The chemic-modified double-strand siRNAs were purchased from GenePharma Co. (Shanghai, P.R. China) after being chemically synthesized, annealed, desalted and purified. The sense strand sequences of siRNAs were as follows: si-Survivin, 5'-AAGGCUGGGAGCCAGAUGACG TT-3' (target site: 315-335) (13); si-XIAP, 5'-GGAGAUACC GUGCGGUGCUTT-3' (target site: 300-318) (14), and si-mock, 5'-UUCUCCGAACGUGUCACGUTT-3'. The mock siRNA was used as the nonsilencing negative control.

Transfection plasmid and siRNA. According to the manufacturer's instructions, $2.0 \mu \mathrm{g}$ plasmid or $20 \mathrm{pmol}$ siRNA and $4 \mu 1$ Lipofectamine 2000 were respectively diluted with $100 \mu 1$ OPTI-MEM medium for $5 \mathrm{~min}$. The two dilutions were mixed gently and incubated for $30 \mathrm{~min}$ at room temperature. Cells were plated in a 12-well plate with a concentration of $4 \times 10^{5}$ cells/well in $800 \mu 1$ of growth medium without antibiotics and immediately transfected with the above $200 \mu 1$ plamid/siRNA-Lipofectamine 2000 mixture (15). Cells were harvested after transfection for $48 \mathrm{~h}$, and the effect of gene silencing was examined with Western blotting. 
A.
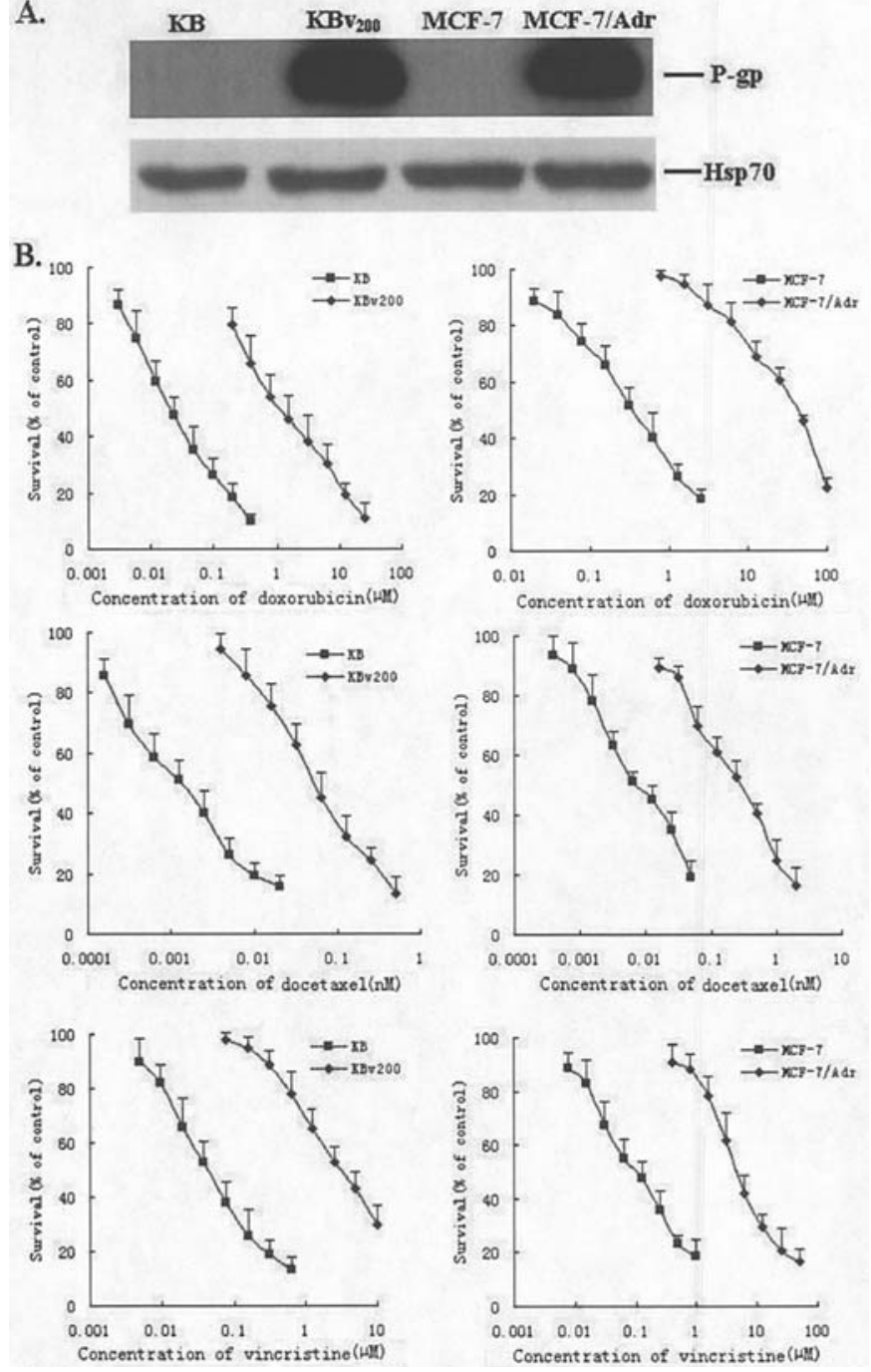

Figure 1. The MDR characteristics of $\mathrm{KBv}_{200}$ and $\mathrm{MCF}-7 / \mathrm{Adr}$ cancer cells (A) P-gp expression was detected with Western blotting in the $\mathrm{KB}, \mathrm{KBv}_{200}$, MCF-7 and MCF-7/Adr cells. Cell lysates were prepared as described in Materials and methods. Equal amounts (100 $\mu \mathrm{g}$ proteins) of cell lysates were separated by SDS-PAGE, and then transferred onto PVDF membrane. The membranes were immunoblotted with primary antibodies including anti-P-gp and anti-Hsp70 at 1:500 dilution. The expression of Hsp70 was used as loading control. The membranes were then incubated for $1 \mathrm{~h}$ with HRPconjugated secondary antibody at 1:1000 dilution. Protein-antibody complex was detected by chemiluminescence. Measurements were performed at least three times and a representative experiment is shown (A). (B) The growth curve of $\mathrm{KB}, \mathrm{KBv}_{200}, \mathrm{MCF}-7$ and $\mathrm{MCF}-7 / \mathrm{Adr}$ cells treated with different concentrations of doxorubicin, docetaxel and vincristine. The inhibition of cell growth was evaluated with the MTT assay as described in Materials and methods. The concentrations required to inhibit growth by $50 \%\left(\mathrm{IC}_{50}\right.$ values) were calculated from cytotoxicity curves by Bliss method. Data points are the means \pm SD of triplicate determinations. Representative experiments are shown.

Western blot analysis. Cells were harvested and rinsed twice with PBS. Cell extracts were prepared with RIPA buffer (1X PBS, $1 \%$ Nonidet P-40, $0.5 \%$ sodium deoxycholate, $0.1 \%$ SDS, $100 \mu \mathrm{g} / \mathrm{ml}$ phenylmethylsulfonyl fluoride, $10 \mu \mathrm{g} / \mathrm{ml}$ aprotinin, $10 \mu \mathrm{g} / \mathrm{ml}$ leupeptin) for $30 \mathrm{~min}$ with occasional rocking and clarified by centrifugation at $12,000 \mathrm{x} \mathrm{g}$ for $15 \mathrm{~min}$ at $4^{\circ} \mathrm{C}$. Identical amounts ( $100 \mu \mathrm{g}$ of protein) of cell lysates were boiled for $10 \mathrm{~min}$ and resolved by sodium dodecyl sulfate polyacrylamide gel electrophoresis (SDS-PAGE) and electrophoretically transferred onto polyvinylidene fluoride (PVDF) membranes. After being incubated in blocking solution containing 5\% powered milk in TBST buffer [10 mM Tris$\mathrm{HCl}(\mathrm{pH} 8.0), 150 \mathrm{mM} \mathrm{NaCl}$, and $0.1 \%$ Tween 20] for $1 \mathrm{~h}$ at $4^{\circ} \mathrm{C}$, the membranes were immunoblotted for $2 \mathrm{~h}$ with primary antibodies including anti-P-gp, anti-Survivin, anti-XIAP and anti-Hsp70 at 1:500 dilution. The expression of Hsp70 was used as loading control. The membranes were then incubated for $1 \mathrm{~h}$ with HRP-conjugated secondary antibody at 1:1000 dilution. Protein-antibody complex was detected by chemiluminescence. Protein expression was quantified by Scion Image software (Scion Co, USA) $(16,17)$.

Immunoprecipitation assay. Protein preparations were similar as for Western blotting, $1.0 \mathrm{mg}$ of total protein was incubated with $10 \mu 1$ of control IgG or anti-P-gp, anti-Survivin or antiXIAP antibody overnight at $4{ }^{\circ} \mathrm{C}$ with gentle rocking after adjusting the volume to $1.0 \mathrm{ml}$ with ice-cold RIPA buffer. Then $20 \mu \mathrm{l}$ protein $\mathrm{A} / \mathrm{G}$ plus-agarose was added to the complex with incubation at $4^{\circ} \mathrm{C}$ for $2 \mathrm{~h}$ on the rocker platform. The immune complex was collected by centrifugation at $1,000 \mathrm{x} \mathrm{g}$ for $30 \mathrm{sec}$ at $4^{\circ} \mathrm{C}$ and washed three times with PBS, and then resuspended with sample loading buffer and boiled for $10 \mathrm{~min}$ and resolved by SDS-PAGE and electrotransferred onto PVDF membrane as previously mentioned. The membrane was incubated and immunoblotted with anti-P-gp, anti-Survivin or anti-XIAP antibody. Protein-antibody complex was detected by chemiluminescence.

Statistical analysis. All experiments were repeated at least three times and differences were determined by using the Student's t-test. Significance was determined at $\mathrm{P}<0.05$.

\section{Results}

The MDR characteristics of $K B v_{200}$ and MCF-7/Adr cancer cell lines. To explore the MDR characteristics of $\mathrm{KBv}_{200}$ and $\mathrm{MCF}-7 / \mathrm{Adr}$ cancer cells, we detected the P-gp expression levels with Western blotting in MDR cancer cells $\mathrm{KBv}_{200}$, $\mathrm{MCF}-7 / \mathrm{Adr}$ and their parental sensitive cells KB and MCF-7, and the cytotoxic effect of several chemotherapeutic agents to these cells with MTT assays. As determined by immunoblot analysis, the membranes of the two MDR cancer cells $\mathrm{KBV}_{200}$ and $\mathrm{MCF}-7 / \mathrm{Adr}$ were a rich source of $\mathrm{P}-\mathrm{gp}$, however, the membranes of their parental sensitive cells $\mathrm{KB}$ and MCF-7 did not detect P-gp (Fig. 1A). Meanwhile, KBv200 cells showed 53.0-, 52.8- and 70.0-fold resistance to doxorubicin, docetaxel and vincristine respectively compared with $\mathrm{KB}$ cells, and MCF-7/Adr cells exhibited 95.3-, 29.8- and 51.4-fold resistance to doxorubicin, docetaxel and vincristine respectively compared with MCF-7 cells (Fig. 1B and Table I). These data suggested that $\mathrm{KBV}_{200}$ and $\mathrm{MCF}-7 / \mathrm{Adr}$ cancer cells possessed the classic MDR phenotypes: overexpression of P-gp and cross-resistance to functionally and structurally unrelated drugs.

$K B v_{200}$ and MCF-7/Adr overexpressed Survivin and XIAP in comparison with their parental sensitive cells. In an attempt to investigate whether the expression levels of IAP family members in MDR cancer cells were different from their parental sensitive cells, we chose the two most important and 
Table I. Cytotoxicity of doxorubicin, docetaxel and vincristine in $\mathrm{KB}, \mathrm{KBv}_{200}, \mathrm{MCF}-7$ and MCF-7/Adr cells.

\begin{tabular}{lcccrrr}
\hline & \multicolumn{5}{c}{$\mathrm{IC}_{50} \pm \mathrm{SD}^{\mathrm{a}}$} \\
\cline { 2 - 7 } Agents & $\mathrm{KB}$ & $\mathrm{KBv}_{200}$ & $\mathrm{FR}$ & $\mathrm{MCF}-7$ & $\mathrm{MCF}-7 / \mathrm{Adr}$ & $\mathrm{FR}$ \\
\hline Doxorubicin $(\mu \mathrm{M})$ & $0.023 \pm 0.002^{\mathrm{b}}$ & $1.22 \pm 0.05$ & $53.0^{\mathrm{c}}$ & $0.334 \pm 0.022$ & $31.82 \pm 3.80$ & 95.3 \\
Docetaxel $(\mathrm{nM})$ & $1.2 \pm 0.2$ & $63.0 \pm 5.0$ & 52.5 & $8.4 \pm 0.05$ & $250.0 \pm 36.0$ & 29.8 \\
Vincristine $(\mu \mathrm{M})$ & $0.045 \pm 0.006$ & $3.15 \pm 0.18$ & 70.0 & $0.106 \pm 0.014$ & $5.45 \pm 0.35$ & 51.4 \\
\hline
\end{tabular}

${ }^{a}$ Cell survival was determined by MTT assay as described in Materials and methods. ${ }^{b}$ Data are means \pm SD of at least three independent experiments performed in triplicate. ${ }^{c} \mathrm{FR}$ (fold-resistance) was the $\mathrm{IC}_{50}$ value of the resistant cell line divided by the $\mathrm{IC}_{50}$ of the parental sensitive cell line.

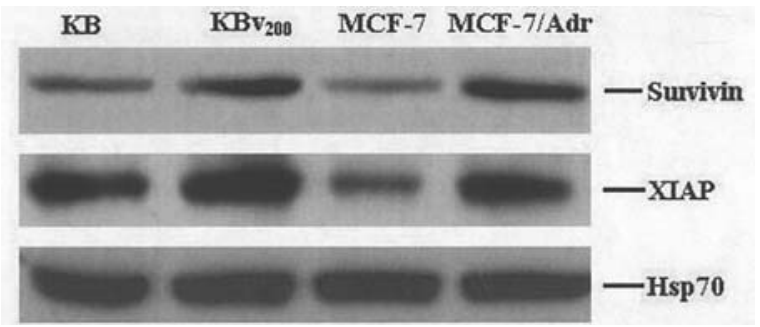

Figure 2. Immunoblot detection of Survivin and XIAP in $\mathrm{KB}, \mathrm{KBv}_{200}$, MCF-7 and MCF-7/Adr cells. Cell lysates from KB, $\mathrm{KBv}_{200}$, MCF-7 and MCF-7/ Adr cells were separated by SDS-PAGE and then transferred onto PVDF membrane as described in Materials and methods. The membranes were immunoblotted with primary antibodies including anti-Survivin, anti-XIAP and anti-Hsp70 at 1:500 dilution. Measurements were performed at least three times and a representative experiment is shown.

well-studied IAP members, Survivin and XIAP, and detected their protein expression levels in the $\mathrm{KBv}_{200}$ and $\mathrm{MCF}-7 / \mathrm{Adr}$ cells and their parental sensitive cells KB and MCF-7 by using Western blot analysis. As shown in Fig. 2, the protein level of Survivin in $\mathrm{KBv}_{200}$ cells was a little higher than that in $\mathrm{KB}$ cells, whereas the protein level of XIAP in $\mathrm{KBv}_{200}$ cells was much higher than that in KB cells. Similarly, MCF-7/ Adr cells showed a slightly high protein level of Survivin and significantly high protein level of XIAP in comparison with MCF-7 cells. These results suggested that in contrast to their parental sensitive cells, MDR cancer cells overexpressed the protein levels of Survivin and XIAP, which were least expressed in $\mathrm{KBv}_{200}$ and MCF-7/Adr cells, and that cancer cells giving rise to MDR were at least in part associated with the overexpression of Survivin and XIAP.

Neither upregulation nor downregulation of Survivin or $X I A P$ affects the expression of $P$-gp. As the expression levels of P-gp, Survivin and XIAP were high in MDR cancer cells, we explored whether the expression change of Survivin or XIAP could have an effect on the P-gp expression. To address this possibility, we used two strategies. Firstly, we upregulated the expression of Survivin or XIAP by transfection with the plasmids which coded the Survivin or XIAP gene. After being transfected with the plasmid pECFPN1-Survivin which expressed the ECFP-Survivin fusion protein, $\mathrm{KB}$ and $\mathrm{KBV}_{200}$

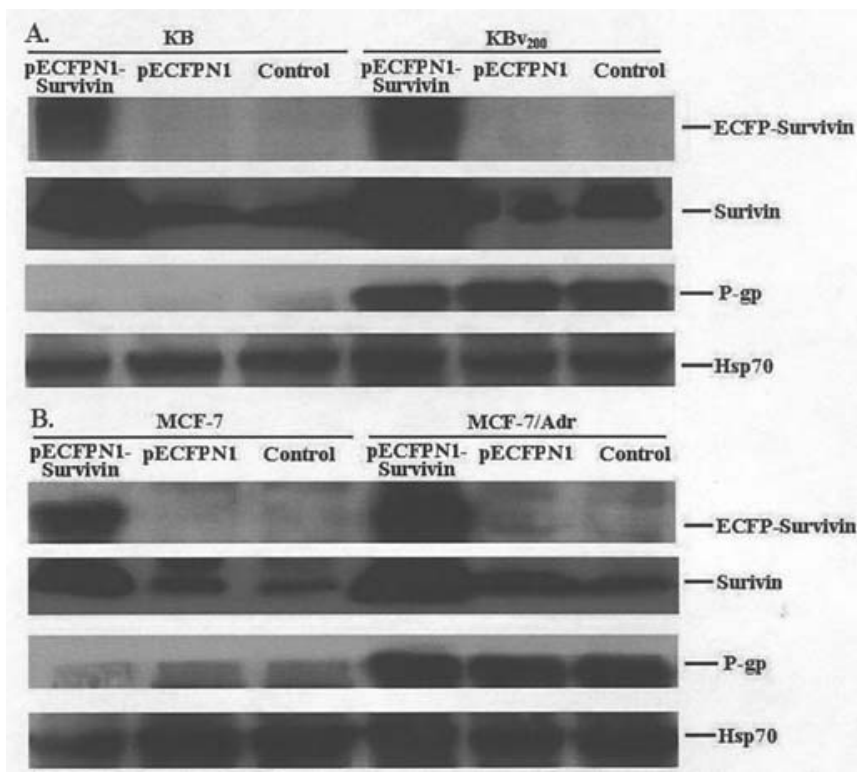

Figure 3. Upregulation of Survivin did not affect the expression of P-gp. Cell lysates were prepared as described in Materials and methods. The protein expression of P-gp and Survivin was detected with Western blotting after transfection for $48 \mathrm{~h}$. The following experiments were the same as described for Fig. 1A. The membranes were immunoblotted with primary antibodies including anti-P-gp, anti-Survivin and anti-Hsp70 at 1:500 dilution. (A) KB and $\mathrm{KBv}_{200}$ cells were transfected with the plasmids pECFPN1-Survivin and pECFPN1. (B) MCF-7 and MCF-7/Adr cells were transfected with the plasmids $\mathrm{pECFPN1-Survivin} \mathrm{and} \mathrm{pECFPN1}$. The protein relative expression level of Survivin in the control group was defined with 1.00 , and other values expressed the protein relative expression level in other groups. Measurements were performed at least three times and a representative experiment is shown. ${ }^{*} \mathrm{P}<0.05$ compared with the corresponding control group.

cells showed increased expression of Survivin protein and ECFP-Survivin fusion protein. The Survivin protein expression of $\mathrm{KB}$ and $\mathrm{KBv}_{200}$ cells was not altered in the empty plasmid pECFPN1 transfected group. However, after transfection with pECFPN1-Survivin, KB cells did not show expression of $\mathrm{P}$-gp, and $\mathrm{KBV}_{200}$ cells did not show increased or decreased expression of P-gp (Fig. 3A). MCF-7 and MCF-7/Adr cells also showed increased expression of Survivin protein and ECFP-Survivin fusion protein following transfection with pECFPN1-Survivin, but MCF-7 cells did not express P-gp 

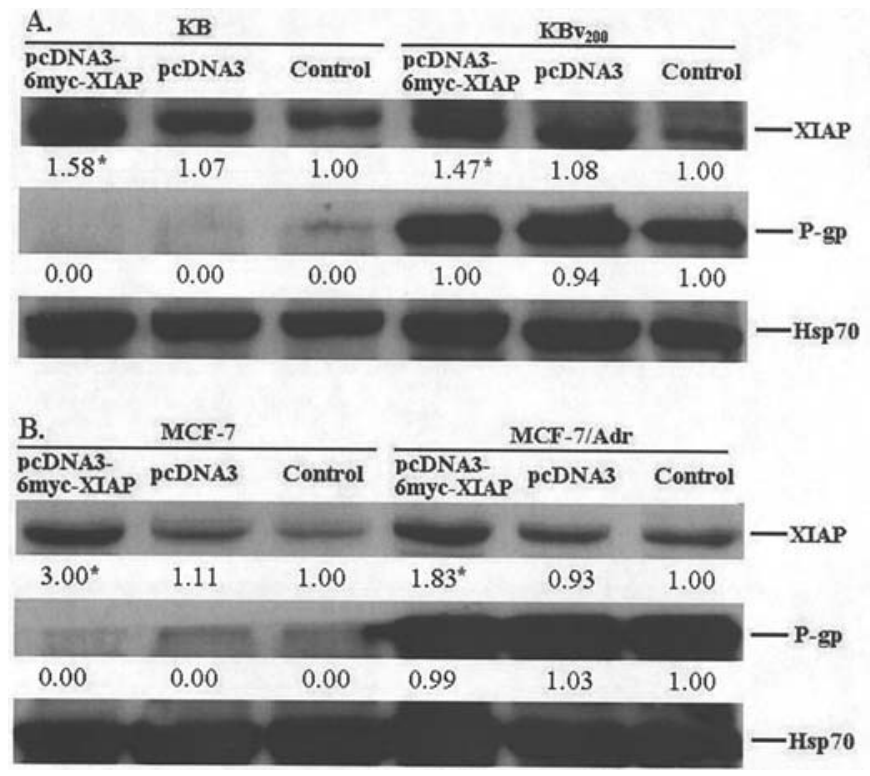

Figure 4. Upregulation of XIAP did not affect the expression of P-gp. Cell lysates were prepared as described in Materials and methods. The protein expression of P-gp and XIAP was detected with Western blotting after transfection for $48 \mathrm{~h}$. The following experiments were the same as described for Fig. 1A. The membranes were immunoblotted with primary antibodies including anti-P-gp, anti-XIAP and anti-Hsp70 at 1:500 dilution. (A) KB and $\mathrm{KBv}_{200}$ cells were transfected with the plasmids pcDNA3-6myc-XIAP and pcDNA3. (B) MCF-7 and MCF-7/Adr cells were transfected with the plasmids pcDNA3-6myc-XIAP and pcDNA3. The protein relative expression level of XIAP in the control group was defined with 1.00, and other values expressed the protein relative expression level in other groups. Measurements were performed at least three times and a representative experiment is shown. ${ }^{*} \mathrm{P}<0.05$ compared with the corresponding control group.
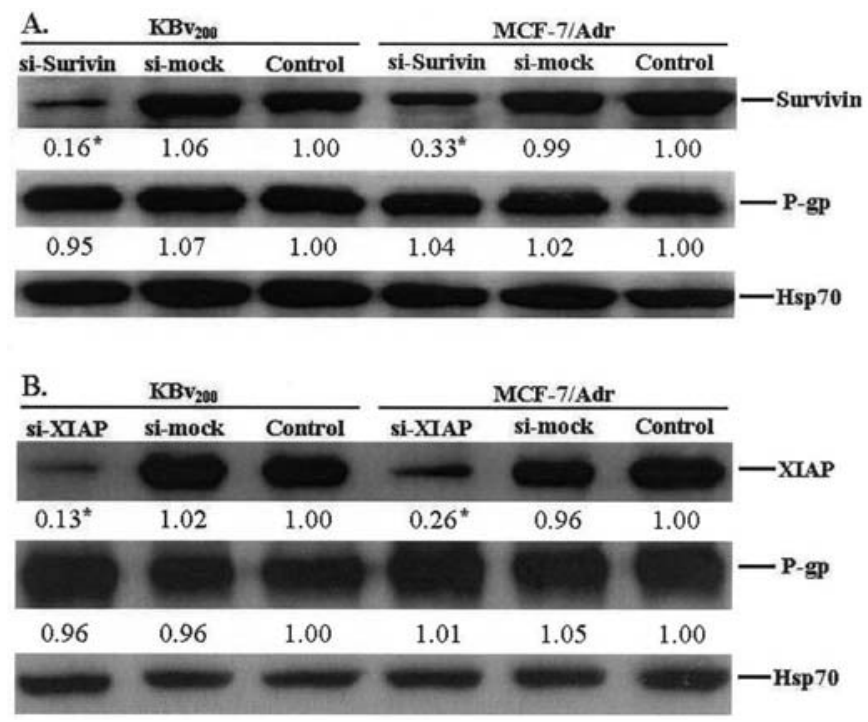

Figure 5. Downregulation of Survivin or XIAP expression did not affect the expression of P-gp. Cell lysates were prepared as described in Materials and methods. The protein expression of P-gp and Survivin was detected with Western blotting after transfection for $48 \mathrm{~h}$. The following experiments were the same as described for Fig. 1A. The membranes were immunoblotted with primary antibodies including anti-P-gp, anti-Survivin or anti-XIAP, and antiHsp70 at 1:500 dilution. (A) $\mathrm{KBv}_{200}$ and MCF-7/Adr cells were transfected with the Survivin siRNA or mock siRNA. (B) MCF-7 and MCF-7/Adr cells were transfected with the XIAP siRNA or mock siRNA. The protein relative expression level of Survivin or XIAP in the control group was defined with 1.00 , and other values expressed the protein relative expression level in other groups. Measurements were performed at least three times and a representative experiment is shown. ${ }^{*} \mathrm{P}<0.05$ compared with the corresponding control group.

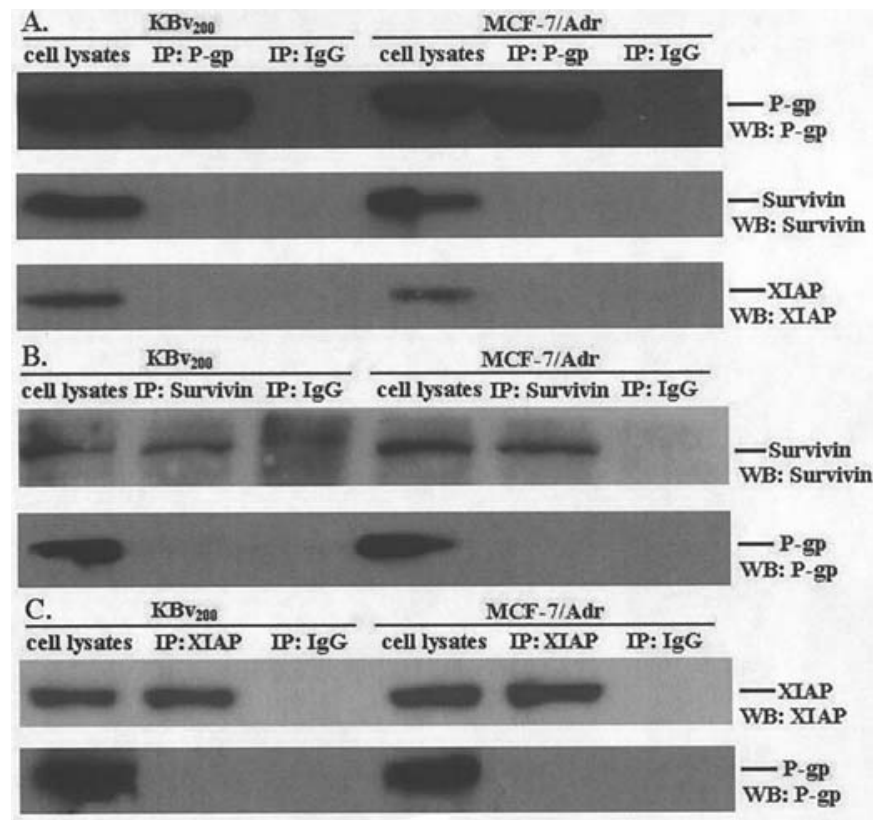

Figure 6. Survivin or XIAP did not directly interact with P-gp. Cell lysates were prepared as described in Materials and methods. Ten mg of total protein was incubated with $10 \mu 1$ of control IgG, anti-P-gp, anti-Survivin or anti-XIAP antibody overnight at $4{ }^{\circ} \mathrm{C}$ with gentle rocking after adjusting the volume to $1.0 \mathrm{ml}$ with ice-cold RIPA buffer. Then $20 \mu 1$ protein A/G plusagarose was added to the complex with incubation at $4^{\circ} \mathrm{C}$ on the rocker platform for $2 \mathrm{~h}$. The immune complex was collected by centrifugation at $1,000 \mathrm{x} \mathrm{g}$ for $30 \mathrm{sec}$ at $4^{\circ} \mathrm{C}$ and washed three times with PBS, then resuspended with sample loading buffer and boiled for $10 \mathrm{~min}$, resolved by SDS-PAGE and electrotransferred onto PVDF membrane as previously described under the Materials and methods. The membrane was incubated and immunoblotted with anti-P-gp, anti-Survivin or anti-XIAP antibody. Protein-antibody complex was detected by chemiluminescence. (A) $\mathrm{KBv}_{200}$ and MCF-7/Adr cell lysates were immunoprecipitated with the control $\mathrm{IgG}$ or anti-P-gp antibody. The immune complexes were detected by using Western blotting with anti-P-gp, anti-Survivin or anti-XIAP antibody. (B) $\mathrm{KBv}_{200}$ and MCF-7/Adr cell lysates were immunoprecipitated with the control IgG or anti-Survivin antibody. The immune complexes were detected by using Western blotting with anti-P-gp or anti-Survivin antibody. (C) $\mathrm{KBv}_{200}$ and MCF-7/Adr cell lysates were immunoprecipitated with the control IgG or anti-XIAP antibody. The immune complexes were detected by using Western blotting with anti-P-gp or anti-XIAP antibody. Measurements were performed at least three times and representative experiments are shown.

and MCF-7/Adr cells did not show altered expression of P-gp (Fig. 3B). Similar results were obtained after transfection with the plasmid pcDNA3-6myc-XIAP which expressed the XIAP protein. In the pcDNA3-6myc-XIAP transfected group, $\mathrm{KB}, \mathrm{MCF}-7, \mathrm{KBV}_{200}$ and MCF-7/Adr cells exhibited increased protein expression of XIAP, but the expression level of P-gp still was not detectable in $\mathrm{KB}$ and MCF-7 cell lines and the expression level of $\mathrm{P}$-gp was not altered in $\mathrm{KBv}_{200}$ and MCF7/Adr cells. In the empty pcDNA3 transfected group, the expression levels of XIAP in all four cells were not changed (Fig. 4A and B).

Secondly, we knocked down the expression of Survivin or XIAP by using transfection with the Survivin or XIAP siRNA to investigate the effect on the expression of P-gp. As shown in Fig. $5 \mathrm{~A}$, both $\mathrm{KBv}_{200}$ and MCF-7/Adr cells showed significantly decreased protein levels of Survivin and unchanged expression of P-gp following transfection with the Survivin siRNA. Likewise, after transfection with the XIAP siRNA, both $\mathrm{KBv}_{200}$ and MCF-7/Adr cells exhibited 
decreased protein levels of XIAP and unchanged expression of P-gp (Fig. 5B). Moreover in the mock siRNA transfected group, neither the protein expression of Survivin nor XIAP in $\mathrm{KBv}_{200}$ and $\mathrm{MCF}-7 / \mathrm{Adr}$ cells was changed. Taken together, these experiments revealed that the increase or decrease of Survivin or XIAP expression did not affect the expression of $\mathrm{P}$-gp, and that the overexpression of P-gp leading to MDR in cancer cells was independent of the overexpression of Survivin or XIAP.

Survivin or XIAP did not directly bind to P-gp. Although we have shown that changing Survivin or XIAP expression did not affect the expression of P-gp, it was necessary to explore the possibility of a physical interaction between P-gp and Survivin or XIAP. The immunoprecipitation assay was used to investigate this issue. We firstly immunoprecipitated P-gp from the cell lysates of $\mathrm{KBv}_{200}$ and MCF-7/Adr cells with the anti-P-gp antibody, and immunoblotted the immunoprecipitation complex with anti-P-gp, anti-Survivin and antiXIAP antibodies. The cell lysates of $\mathrm{KBv}_{200}$ and MCF-7/Adr cells were used as the control to confirm the validity of immunoblotting. As shown in Fig. 6A, there was only P-gp in the immunoprecipitation complex formed with anti-P-gp antibody, not Survivin or XIAP, and neither P-gp, Survivin nor XIAP was contained in the control IgG immunoprecipitation complex. On the other hand, we immunoprecipitated Survivin or XIAP protein from the cell lysates of $\mathrm{KBv}_{200}$ and MCF-7/ Adr cells with the anti-Survivin or anti-XIAP antibodies. As a result, there was only Survivin and no P-gp in the immunoprecipitation complex formed with the anti-Survivin antibody (Fig. 6B). Similary, there was XIAP but no P-gp contained in the anti-XIAP antibody immunoprecipitation complex (Fig. 6C). Therefore, these data indicated that neither Survivin nor XIAP directly interacted with or bound to P-gp.

\section{Discussion}

In the present study, our results showed that the two classic MDR cancer cells $\mathrm{KBv}_{200}$ and MCF-7/Adr overexpressed high protein levels of P-gp, XIAP and Survivin, and conferred high levels of resistance to chemotherapeutic drugs doxorubicin, docetaxel and vincristine in contrast to their parental sensitive $\mathrm{KB}$ and MCF-7 cells. This result was consistent with another report which revealed that MDR cancer cells, HL-60R with high expression of P-gp, significantly overexpressed both the mRNAs and the IAPs including c-IAP1, c-IAP2, XIAP, NAIP and Survivin in comparison with those of the parent sensitive HL-60 cells, except for c-IAP-1 mRNA (13), However, the other study examined the DNA-binding capacity of NF- $\mathrm{KB}$ (p50 or p65 subunits) in HL-60 and HL-60R cells and found that HL-60 cells expressed only constitutive activation of p50, while HL-60R possessed the activated forms of both p50 and p65, suggesting that the constitutively activated p50-p65 heterodimer in HL-60R cells correlates well with their increased levels of IAPs. Nevertheless, IAPs are positively and negatively regulated by very complicated mechanisms. The pro-survival effect of NF- $\mathrm{KB}$ activation is linked to the upregulation of several IAPs, including Survivin, XIAP and c-IAP2 (18-20). Wild-type p53 suppresses Survivin expression, and loss of or mutant p53 results in upregulation of Survivin expression $(21,22)$. Several studies have demonstrated that Survivin is regulated by $\beta$-catenin signaling in colon cancer (23), by ErbB2 and EGFR via PI3K/AKT pathway in breast cancer cells $(24,25)$, and by the Bcr-Abl/MAPK cascade in $\mathrm{Ph}^{+} \mathrm{CML}$ (26). In addition, Stat 3 directly binds to the Survivin promoter and upregulates its expression (27). Oncogenic cH-Ras also upregulates Survivin depending on the functional Ras/PI3-K and Ras/Raf/MEK/MAPK signaling pathways (28). However, it should be noted that any mutations or alterations of the factors or pathways that are involved in regulation of IAPs could result in constitutive upregulation of IAPs, and it is likely that different mechanisms lead to the expression disregulation of IAPs in different cancer cells. Hence, future studies are indispensable to identify which mechanisms result in the overexpression of IAPs in MDR cancer cells.

Cancer cells selected for resistance to chemotherapeutic drugs involved in the MDR phenotype often exhibit constitutive overexpression of P-gp. Although frequently it is the result of MDR1 gene amplification, this overexpression can also be mediated at the level of transcription. Various factors or pathways play a role in regulation of P-gp expression. Wildtype $\mathrm{p} 53$ represses transcription of the MDR1 gene, however, mutant $\mathrm{p} 53$ proteins are able to activate the MDR1 promoter $(29,30)$. The MDR1 gene is also a target of the Ras/Raf signaling pathway (31). The TCF4/ß-catenin complex has been reported as an MDR1 gene transcriptional activator that may promote early abnormal expression of $\mathrm{P}$-gp in colorectal carcinoma (32). It has been reported previously that the promoter of the MDR1 gene possesses a negative binding site of AP-1 (c-Jun/c-Fos) (33), and that P-gp downregulation requires the catalytic activity of JNK and is mediated by the c-Jun transcription factor (34). Additionally, cross-coupled $\mathrm{NF}-\kappa \mathrm{B} / \mathrm{p} 65$ and c-Fos complex has been reported to negatively regulate the promoter activity of the MDR1 gene (35). The transcription factor HSF1, Sp1 and the Y-box binding protein YB1 have been shown to positively regulate P-gp expression (36-38). Another transcription factor NF-Y mediates the regulation of the MDR1 gene by histone acetyltransferase and deacetylase (39). From these studies of identifying the mechanisms of regulating the expression of P-gp and IAPs, we can deduce that some factors or pathways, such as $\mathrm{p} 53$, $\mathrm{NF}-\kappa \mathrm{B}, \mathrm{B}$-catenin and Ras/Raf signaling pathway, may simultaneously regulate the expression of P-gp and IAPs. Furthermore, several studies have reported that these factors or pathways are able to simultaneously regulate P-gp and IAP expression could also be conversely affected by IAPs. For example, Survivin regulates p53 expression and modifies p53 degradation through a caspase-3/Mdm2 axis that is Survivin-mediated inhibition of Mdm2 cleavage by caspases (40). XIAP inhibits JNK1 activation by TGF-ß1 through ubiquitin-mediated proteosomal degradation of the TAK1 (41). Many IAPs have the RING domain which can recruit E2 ubiquitin-conjugating enzymes and catalyse the transfer of ubiquitin onto target proteins (42). The stability and function of P-gp can be regulated by the ubiquitin-proteasome pathway (43). According to this evidence, we presumed that IAPs could regulate the expression of P-gp through some factors or pathways as mentioned above or other unknown mechanisms. Therefore, subsequently we used the gene-transfer technology 
to verify our speculation. At first, we transfected the two sensitive cell lines KB and MCF-7 which do not express P-gp with the plasmids pECFPN1-Survivin or pcDNA3-6mycXIAP to upregulate the expression of Survivin or XIAP and to observe whether P-gp expression follows transfection. However, our results suggest that neither Survivin nor XIAP upregulation can regulate the $\mathrm{P}$-gp expression of both $\mathrm{KB}$ and MCF-7 cell lines. Secondly, we transfected the two MDR cells $\mathrm{KBv}_{200}$ and MCF-7/Adr which overexpress P-gp with the same plasmids of pECFPN1-Survivin or pcDNA3-6mycXIAP to observe whether after transfection, P-gp expression could be altered. Our experimental results were coincident that P-gp expression in both cell lines was unchanged. From these two experiments, we conclude that upregulating the expression of either Survivin or XIAP does not affect the expression of P-gp. Thirdly, we used RNAi strategies to downregulate Survivin or XIAP expression and to observe the change of $\mathrm{P}$-gp expression in $\mathrm{KBv}_{200}$ and MCF-7/Adr cells. Surprisingly, these two MDR cell lines showed unchanged P-gp expression after transfection with Survivin or XIAP siRNA and the expression levels of Survivin or XIAP were significantly decreased. In summary, these experimental data indicate that neither Survivin nor XIAP has an effect on the expression of P-gp in cancer cells, at least this was the case for the four cell lines used in this study.

Several studies have proved that functional P-gp can confer resistance to apoptosis induced not only by chemotherapeutical drugs but also by diverse nondrug apoptosis stimuli including serum starvation, UV and $\gamma$ irradiation, Fas and TNF (4-6). One mechanism of this phenomenon is that P-gp may efflux drugs out of the cell as a 'pump' resulting in the significantly low intercellular concentration of drugs, but the nondrug apoptosis stimuli can't be effluxed by P-gp directly. So how P-gp gives rise to resistance to apoptosis induced by these diverse nondrug apoptosis stimuli is worth attention. It has been demonstrated that P-gp could inhibit caspase activation resulting in resistance to apoptosis, and this inhibitory effect could be reversed by using P-gp antagonists $(4,5,44)$. As we know, IAPs can directly bind to and inhibit specific caspase activation (8), however, it is unknown how P-gp affects caspase activation. Therefore, we supposed that P-gp affecting caspase activation could be associated with IAPs and there was the possibility of a physical interaction between Survivin or XIAP and P-gp. We used the immunoprecipitation assay to validate this supposition. $\mathrm{P}$-gp was immunoprecipitated from the cell lysates of $\mathrm{KBv}_{200}$ and MCF-7/Adr cells with the anti-P-gp antibody, and immunoblotted with anti-P-gp, anti-Survivin and anti-XIAP antibodies, but after immunoblotting there was only P-gp, no Survivin or XIAP, in the immunoprecipitation complex formed with anti-P-gp antibody. Conversely, we immunoprecipitated Survivin or XIAP protein with the anti-Survivin or antiXIAP antibodies, similarly only Survivin but no P-gp was in the immunoprecipitation complex formed with the antiSurvivin antibody, and only XIAP but no P-gp was contained in the anti-XIAP antibody immunoprecipitation complex. Consequently, these data suggested that there was no physical interaction between Survivin or XIAP and P-gp. It seems that P-gp affecting caspase activation did not correlate with Survivin or XIAP.
In conclusion, our study demonstrated that the two classic MDR cancer cells $\mathrm{KBv}_{200}$ and MCF-7/Adr, which have a very high protein expression level of $\mathrm{P}$-gp as well as high protein expression levels of XIAP and Survivin, conferred very high resistance to chemotherapeutic drugs doxorubicin, docetaxel and vincristine in comparison with their parental sensitive cells $\mathrm{KB}$ and MCF-7 cell lines. Neither upregulation nor downregulation of Survivin or XIAP affected the expression of P-gp in these four cell sublines. We concluded that either Survivin or XIAP does not directly interact with P-gp. The mechanisms of the overexpression of IAPs in MDR cancer cells and P-gp affecting caspase activation need to be studied further.

\section{Acknowledgements}

This study was supported by funds from Chinese Ministry of Education Doctor Foundation no. 20050558062 and Guangdong Provincial Key Sciences Foundation no. 2004B30101005 (LW Fu) and St. John's University Tenure Track Faculty StartUp Funding no. C-0531 (ZS Chen). We thank Dr Marko J. Kallio (VTT-Medical Biotechnology and University of Turku, Finland) for providing the plasmid pECFPN1-Survivin and Dr Robert G. Korneluk (University of Ottawa, Canada) for the gift of plasmid pcDNA3-6myc-XIAP. We also thank Professor YS Liu (Chinese Academy of Medical Sciences, Beijing) and Professor XY Liu (National Cancer Institute, USA) for providing the cell lines for this study. Z Shi is a recipient of Kaisi fellowship for overseas study at St. John's University from Sun Yat-Sen University. We thank the staff and students of the Cancer Chemotherapy laboratory (ZS Chen) for helpful discussions and critical reading of the manuscript.

\section{References}

1. Gottesman MM, Fojo T and Bates SE: Multidrug resistance in cancer: role of ATP-dependent transporters. Nat Rev Cancer 2: 48-58, 2002.

2. Gottesman MM: Mechanisms of cancer drug resistance. Annu Rev Med 53: 615-627, 2002.

3. Kruh GD: Introduction to resistance to anticancer agents. Oncogene 22: 7262-7264, 2003.

4. Smyth MJ, Krasovskis E, Sutton VR and Johnstone RW: The drug efflux protein, P-glycoprotein, additionally protects drugresistant tumor cells from multiple forms of caspase-dependent apoptosis. Proc Natl Acad Sci USA 95: 7024-7029, 1998.

5. Johnstone RW, Cretney E and Smyth MJ: P-glycoprotein protects leukemia cells against caspase-dependent, but not caspaseindependent, cell death. Blood 93: 1075-1085, 1999.

6. Ruth AC and Roninson IB: Effects of the multidrug transporter $\mathrm{P}$-glycoprotein on cellular responses to ionizing radiation. Cancer Res 60: 2576-2578, 2000.

7. Nachmias B, Ashhab Y and Ben-Yehuda D: The inhibitor of apoptosis protein family (IAPs): an emerging therapeutic target in cancer. Semin Cancer Biol 14: 231-243, 2004.

8. Vaux DL and Silke J: IAPs - the ubiquitin connection. Cell Death Differ 12: 1205-1207, 2005.

9. Altieri DC: Survivin, versatile modulation of cell division and apoptosis in cancer. Oncogene 22: 8581-8589, 2003.

10. Rothe M, Pan MG, Henzel WJ, Ayres TM and Goeddel DV: The TNFR2-TRAF signaling complex contains two novel proteins related to baculoviral inhibitor of apoptosis proteins. Cell 83: 1243-1252, 1995.

11. Notarbartolo M, Cervello M, Dusonchet L, Cusimano A and D'Alessandro N: Resistance to diverse apoptotic triggers in multidrug resistant HL60 cells and its possible relationship to the expression of P-glycoprotein, Fas and of the novel antiapoptosis factors IAP (inhibitory of apoptosis proteins). Cancer Lett 180: 91-101, 2002. 
12. Notarbartolo M, Cervello M, Poma P, et al: Expression of the IAPs in multidrug resistant tumor cells. Oncol Rep 11: 133-136, 2004.

13. Lima RT, Martins LM, Guimaraes JE, Sambade C and Vasconcelos MH: Specific downregulation of bcl-2 and xIAP by RNAi enhances the effects of chemotherapeutic agents in MCF-7 human breast cancer cells. Cancer Gene Ther 11: 309-316, 2004.

14. Chawla-Sarkar M, Bae SI, Reu FJ, et al: Downregulation of Bcl-2, FLIP or IAPs (XIAP and survivin) by siRNAs sensitizes resistant melanoma cells to Apo2L/TRAIL-induced apoptosis. Cell Death Differ 11: 915-923, 2004.

15. Amarzguioui M: Improved siRNA-mediated silencing in refractory adherent cell lines by detachment and transfection in suspension. Biotechniques 36: 766-768, 2004.

16. Shi Z, Liang YJ, Chen ZS, et al: Reversal of MDR1/Pglycoprotein-mediated multidrug resistance by vector-based RNA interference in vitro and in vivo. Cancer Biol Ther 5: 39-47, 2006.

17. Wang XW, Ding Y, Liang YJ, et al: Enhancement of FG020326 on sensitivity of MCF-7/ADR cells to taxotere via enhancing activation of caspase- 8 and caspase-3. Ai Zheng 23: 1379-1385, 2004.

18. Chu ZL, McKinsey TA, Liu L, et al: Suppression of tumor necrosis factor-induced cell death by inhibitor of apoptosis c-IAP2 is under NF-kappaB control. Proc Natl Acad Sci USA 94: 10057-10062, 1997.

19. Stehlik C, de Martin R, Kumabashiri I, et al: Nuclear factor (NF)kappaB-regulated X-chromosome-linked iap gene expression protects endothelial cells from tumor necrosis factor alphainduced apoptosis. J Exp Med 188: 211-216, 1998.

20. Kawakami H, Tomita M, Matsuda T, et al: Transcriptional activation of survivin through the NF-kappaB pathway by human T-cell leukemia virus type I tax. Int J Cancer 115: 967-974, 2005.

21. Mirza A, McGuirk M, Hockenberry TN, et al: Human survivin is negatively regulated by wild-type p53 and participates in p53dependent apoptotic pathway. Oncogene 21: 2613-2622, 2002.

22. Hoffman WH, Biade S, Zilfou JT, Chen J and Murphy M: Transcriptional repression of the anti-apoptotic survivin gene by wild type p53. J Biol Chem 277: 3247-3257, 2002.

23. Zhang T, Otevrel T, Gao Z, et al: Evidence that APC regulates survivin expression: a possible mechanism contributing to the stem cell origin of colon cancer. Cancer Res 61: 8664-8667, 2001.

24. Asanuma H, Torigoe T, Kamiguchi K, et al: Survivin expression is regulated by coexpression of human epidermal growth factor receptor 2 and epidermal growth factor receptor via phosphatidylinositol 3-kinase/AKT signaling pathway in breast cancer cells. Cancer Res 65: 11018-11025, 2005.

25. Xia W, Bisi J, Strum J, et al: Regulation of survivin by ErbB2 signaling: therapeutic implications for ErbB2-overexpressing breast cancers. Cancer Res 66: 1640-1647, 2006.

26. Carter BZ, Mak DH, Schober WD, et al: Regulation of survivin expression through Bcr-Abl/MAPK cascade: targeting survivin overcomes imatinib resistance and increases imatinib sensitivity in imatinib-responsive CML cells. Blood 107: 1555-1563, 2006.

27. Gritsko T, Williams A, Turkson J, et al: Persistent activation of stat3 signaling induces survivin gene expression and confers resistance to apoptosis in human breast cancer cells. Clin Cancer Res 12: 11-19,2006.

28. Sommer KW, Schamberger CJ, Schmidt GE, Sasgary S and Cerni C: Inhibitor of apoptosis protein (IAP) survivin is upregulated by oncogenic c-H-Ras. Oncogene 22: 4266-4280, 2003.
29. Zastawny RL, Salvino R, Chen J, Benchimol S and Ling V: The core promoter region of the P-glycoprotein gene is sufficient to confer differential responsiveness to wild-type and mutant p53. Oncogene 8: 1529-1535, 1993.

30. Thottassery JV, Zambetti GP, Arimori K, Schuetz EG and Schuetz JD: p53-dependent regulation of MDR1 gene expression causes selective resistance to chemotherapeutic agents. Proc Natl Acad Sci USA 94: 11037-11042, 1997.

31. Kim SH, Lee SH, Kwak NH, Kang CD and Chung BS: Effect of the activated Raf protein kinase on the human multidrug resistance 1 (MDR1) gene promoter. Cancer Lett 98: 199-205, 1996.

32. Yamada T, Takaoka AS, Naishiro Y, et al: Transactivation of the multidrug resistance 1 gene by T-cell factor 4/beta-catenin complex in early colorectal carcinogenesis. Cancer Res 60: 4761-4766, 2000 .

33. Ikeguchi M, Teeter LD, Eckersberg T, Ganapathi R and Kuo MT: Structural and functional analyses of the promoter of the murine multidrug resistance gene $\mathrm{mdr} 3 / \mathrm{mdr} 1 \mathrm{a}$ reveal a negative element containing the AP-1 binding site. DNA Cell Biol 10: 639-649, 1991.

34. Zhou J, Liu M, Aneja R, et al: Reversal of P-glycoproteinmediated multidrug resistance in cancer cells by the c-Jun NH2terminal kinase. Cancer Res 66: 445-452, 2006.

35. Ogretmen B and Safa AR: Negative regulation of MDR1 promoter activity in MCF-7, but not in multidrug resistant MCF-7/Adr, cells by cross-coupled NF-kappa B/p65 and c-Fos transcription factors and their interaction with the CAAT region. Biochemistry 38: 2189-2199, 1999.

36. Vilaboa NE, Galan A, Troyano A, de Blas E and Aller P: Regulation of multidrug resistance 1 (MDR1)/P-glycoprotein gene expression and activity by heat-shock transcription factor 1 (HSF1). J Biol Chem 275: 24970-24976, 2000.

37. Ohga T, Uchiumi T, Makino Y, et al: Direct involvement of the Y-box binding protein YB-1 in genotoxic stress-induced activation of the human multidrug resistance 1 gene. J Biol Chem 273: 5997-6000, 1998.

38. Cornwell MM and Smith DE: SP1 activates the MDR1 promoter through one of two distinct G-rich regions that modulate promoter activity. J Biol Chem 268: 19505-19511, 1993.

39. Jin S and Scotto KW: Transcriptional regulation of the MDR1 gene by histone acetyltransferase and deacetylase is mediated by NF-Y. Mol Cell Biol 18: 4377-4384, 1998.

40. Wang Z, Fukuda S and Pelus LM: Survivin regulates the p53 tumor suppressor gene family. Oncogene 23: 8146-8153, 2004.

41. Kaur S, Wang F, Venkatraman M and Arsura M: X-linked inhibitor of apoptosis (XIAP) inhibits c-Jun N-terminal kinase 1 (JNK1) activation by transforming growth factor beta1 (TGFbeta1) through ubiquitin-mediated proteosomal degradation of the TGF-beta1-activated kinase 1 (TAK1). J Biol Chem 280: 38599-38608, 2005 .

42. Vaux DL and Silke J: IAPs, RINGs and ubiquitylation. Nat Rev Mol Cell Biol 6: 287-297, 2005.

43. Zhang Z, Wu JY, Hait WN and Yang JM: Regulation of the stability of P-glycoprotein by ubiquitination. Mol Pharmacol 66: 395-403, 2004.

44. Ruefli AA, Tainton KM, Darcy PK, Smyth MJ and Johnstone RW: P-glycoprotein inhibits caspase-8 activation but not formation of the death inducing signal complex (disc) following Fas ligation. Cell Death Differ 9: 1266-1272, 2002. 